\title{
HEPATECTOMIA PARCIAL EM CIRURGIA PEDIÁTRICA
}

\section{PARTIAL HEPATECTOMY IN PEDIATRIC SURGERY}

\author{
Roberto Oliveira Cardoso dos Santos ${ }^{1}$ \\ Luis Donizeti da Silva Stracieri, TCBC ${ }^{2}$ \\ Yvone A. M. Villela de Andrade Vicente ${ }^{3}$ \\ José Janeiro Pato Garrido ${ }^{4}$ \\ Flávio de Oliveira Pileggi ${ }^{1}$
}

\begin{abstract}
RESUMO: Objetivo: Embora, atualmente, as indicações de hepatectomias em crianças sejam menos frequentes, em alguns casos elas constituem a melhor opção terapêutica. O objetivo deste trabalho é relatar a experiência de dez anos com grandes ressecções hepáticas em pacientes pediátricos. Método: Foram analisados os dados de doze pacientes submetidos a lobectomia hepática nos serviços de Cirurgia Pediátrica do Hospital da Clínicas da Faculdade de Medicina da Universidade de São Paulo e do Hospital Santa Lydia, em Ribeirão Preto (SP) de 1985 a 1995. Resultados: Foram realizadas oito lobectomias esquerdas e quatro lobectomias direitas. Dez crianças foram operadas por neoplasia e duas por complicações de traumatismo hepático. A idade das dez crianças portadoras de neoplasia variou de seis dias a dezesseis meses, sendo em média 3,8 meses. O diagnóstico histopatológico foi hemangioendotelioma em cinco (50\%), hepatoadenoma em dois (20\%), hepatoblastoma em dois $(20 \%)$ e hepatocarcinoma em um (10\%). O peso do tumor correspondeu em média a 7,1\% do peso do paciente. A duração média da cirurgia foi de 2 horas e 58 minutos. O seguimento pós-operatório variou até 141 meses, sendo em média 76,5 meses. Sete pacientes receberam transfusão de sangue intra-operatória, correspondente a $23,3 \%$ de sua volemia, em média. Um deles apresentou recidiva de tumor, necessitando reoperação. Nenhum dos doze pacientes apresentou complicações pós-operatórias. Conclusão: A hepatectomia parcial é um procedimento difícil tecnicamente, que, no entanto, pode ser realizado com segurança, mesmo em hospitais que não disponham de recursos tecnológicos sofisticados, desde que o cirurgião esteja bem preparado para enfrentar suas dificuldades.
\end{abstract}

Descritores: Hepatectomia; Tumores hepáticos; Crianças

\section{INTRODUÇÃO}

As hepatectomias parciais são procedimentos cirúrgicos de grande porte, muitas vezes necessários para tratamento de neoplasias hepáticas benignas ou malignas, e que podem acarretar índices relativamente elevados de morbidade e mortalidade. Embora atualmente as indicações cirúrgicas de grandes ressecções hepáticas em crianças sejam menos freqüientes, em alguns casos elas ainda são a melhor opção terapêutica. O objetivo deste trabalho é relatar nossa experiência de dez anos com a hepatectomia em pacientes pediátricos e mostrar que é possível realizar uma lobectomia hepática com excelentes

1 Médico Assistente da disciplina de Cirurgia Pediátrica do Hospital das Clínicas da FMUSP-RP

2 Médico Assistente da disciplina de Cirurgia de Urgência do Hospital das Clínicas da FMUSP-RP

3 Professora Doutora da disciplina de Cirurgia Pediátrica do Hospital das Clínicas da FMUSP-RP

4 Médico do Serviço de Cirurgia Pediátrica do Hospital Santa Lydia - RP

Recebido em 30/10/2001

Aceito para publicação em 17/09/2002

Trabalho realizado nos serviços de Cirurgia Pediátrica do Hospital das Clínicas da Faculdade de Medicina da Universidade de São Paulo em Ribeirão Preto, e do Hospital Santa Lydia em Ribeirão Preto. 
resultados, mesmo em centros médicos que não disponham de recursos tecnológicos sofisticados. Para isto é fundamental que o cirurgião atenha-se rigorosamente a uma apurada técnica cirúrgica e conheça profundamente os detalhes anatômicos das estruturas intra e extra-hepáticas ${ }^{1}$.

\section{MÉTODO}

Foram analisados os dados de 12 pacientes submetidos a lobectomia hepática no serviço de Cirurgia Pediátrica do Hospital Santa Lydia e no Hospital das Clínicas da Faculdade de Medicina da Universidade de São Paulo, em Ribeirão Preto, no Estado de São Paulo, de 1985 a 1995.

Foram incluídas no presente estudo todas as crianças portadoras de neoplasias benignas ou malignas submetidas a hepatectomia direita ou esquerda (seguindo-se a nomenclatura proposta por Couinaud ${ }^{2,3}$ ). Foram também incluídas as crianças submetidas a hepatectomia regrada direita ou esquerda por complicações de traumatismo hepático.

Foram excluídas deste estudo as crianças com neoplasias hepáticas benignas nas quais tenha se optado por conduta expectante e que, portanto, não foram submetidas a tratamento cirúrgico. Foram também excluídos os pacientes portadores de neoplasias malignas consideradas irressecáveis por envolverem ambos os lobos do fígado ou apresentarem metástases à distância. Também não foram analisados os casos submetidos à tumorectomia simples, enucleação do tumor, ressecção em cunha de pequenos tumores, bem como os pacientes submetidos a hepatectomias parciais não regradas em casos de traumatismo nos quais se tenha aproveitado o plano de laceração hepática como linha de secção do parênquima.

Dez crianças foram operadas por neoplasia e duas por complicações de traumatismo hepático. Estes dois grupos foram analisados separadamente.

A idade das dez crianças portadoras de neoplasia variou de seis dias a 16 meses, sendo em média 3,8 meses. Apenas um caso (10\%) havia ultrapassado um ano de idade. Sete eram do sexo masculino (70\%) e três do sexo feminino (30\%).

A idade das duas crianças operadas por complicação de traumatismo hepático foi de 10 e 13 anos, ambas do sexo masculino.

O diagnóstico histopatológico da neoplasia hepática foi de hemangioendotelioma em cinco casos
(50\%), hepatoadenoma em dois casos (20\%), hepatoblastoma em dois (20\%), e hepatocarcinoma em um $(10 \%)$. O peso dos tumores ressecados variou de 90 a $740 \mathrm{~g}$, correspondendo em média a $7,1 \%$ do peso total do paciente. Foram realizadas sete lobectomias esquerdas $(70 \%)$ e três lobectomias direitas $(30 \%)$.

\section{RESULTADOS}

Nas dez crianças submetidas a hepatectomia parcial por neoplasia hepática, o volume de transfusão sangüínea intra-operatória variou de 0 a $450 \mathrm{ml}$. Três crianças (30\%) não receberam transfusão de sangue.

A duração do procedimento cirúrgico nestes dez pacientes foi, em média, de 2 horas e 58 minutos, chegando em um caso a 5 horas de cirurgia. A maior dificuldade técnica em se realizar a hepatectomia hepática direita reflete-se no tempo cirúrgico, em média quase uma hora e meia mais longo que nas hepatectomias esquerdas: 2 horas e 39 minutos nestas, e 4 horas e 5 minutos naquelas, em média. Esta diferença deve-se não só ao maior tamanho do lobo direito (e dos tumores nele localizados), mas também a mais complexos problemas técnicos envolvendo dissecção da veia cava inferior, ligadura individualizada de ramos venosos que saem diretamente do lobo direito para a veia cava, bem como à maior dificuldade de acesso à face convexa do lobo direito hepático (Figura-1).

Houve também diferença significativa no volume transfundido entre os casos de hepatectomia direita e esquerda. Os pacientes com neoplasia do lobo hepático direito receberam, em média, transfusão correspondente a $38,5 \%$ de sua volemia, enquanto que as crianças portadoras de tumor do lobo esquerdo do fígado receberam em média o equivalente a $16,8 \%$ de sua volemia.

As duas crianças submetidas a ressecção hepática por causas não neoplásicas eram casos complicados que apresentaram evolução atípica após traumatismos graves de fígado, não podendo portanto ser comparadas às crianças em que a lobectomia foi realizada de forma programada por serem portadoras de tumores hepáticos. Ambos eram do sexo masculino, com idade de 10 e 13 anos. A cirurgia nestes dois casos durou em média 5 horas e 30 minutos, e receberam um volume de transfusão sangüínea correspondente a $74,2 \%$ de sua volemia. 
Um deles foi submetido a hepatectomia esquerda em caráter de emergência, poucas horas após sofrer o trauma; o outro foi submetido à hepatectomia direita eletiva por seqüela de traumatismo cerca de dois meses após o acidente. Este paciente era portador de hemobilia, com três volumosas cavidades ocupando grande parte do lobo direito do fígado, como sequiela de um traumatismo hepático prévio (Figura2 ). A hemorragia através das vias biliares era freqüente e considerável, não respondendo às medidas conservadoras, menos agressivas, até que por fim, como último recurso, optou-se por hepatectomia direita, com resolução dos sintomas. Este paciente foi o único desta casuística em que foi necessária uma abordagem por tóraco-laparotomia.

O seguimento pós-operatório nos pacientes operados por neoplasia variou até 141 meses, sendo, em média, de 76,5 meses. Uma das crianças portadoras de hepatoblastoma apresentou recidiva local duas vezes, tendo sido nestas ocasiões reoperada para nova ressecção da massa tumoral. Em ambas as ocasiões a indicação cirúrgica foi a presença de massa aparentemente localizada e ressecável (aos exames de imagem) e não responsiva à quimioterapia. Entretanto, evoluiu com má resposta à terapêutica instituída, com óbito após alguns meses de seguimento.

Os outros nove pacientes apresentaram boa evolução do ponto de vista oncológico, e estão vivos, sem evidência de doença. Nenhum deles apresentou qualquer sinal clínico ou laboratorial de comprometimento da função hepática. Em nenhum caso houve abscesso, hematoma, fístula, ou qualquer outra complicação importante no período pós-operatório.

Entre as outras duas crianças operadas por causas não neoplásicas, uma foi reoperada após 8 dias para correção de obstrução duodenal causada por um grande hematoma mural secundário ao mesmo traumatismo que acarretou a lesão hepática, e que não regrediu, persistindo com obstrução da luz duodenal. Estas duas crianças apresentaram boa evolução a longo prazo, sem qualquer outra complicação cirúrgica.

\section{DISCUSSÃO}

As hepatectomias parciais são cirurgias pouco freqüentes na faixa etária pediátrica, e o cirurgião deve conhecer muito bem suas indicações e contraindicações. As lobectomias hepáticas em cirurgia pediátrica são indicadas principalmente para o tratamento dos tumores malignos primários do fígado, dos quais o mais freqüente é o hepatoblastoma, e para tumores benignos cujo tamanho seja tão grande que cause compressão de órgãos vizinhos, ou dificuldade respiratória por elevação da cúpula diafragmática. Tumores benignos pequenos podem ser apenas enucleados, se estiverem situados em região central do fígado, ou sua exérese pode ser feita através de ressecção em cunha quando forem mais periféricos. Em alguns casos, se houver certeza de que o tumor é benigno, e não houver crescimento exagerado da massa tumoral, pode-se acompanhar clinicamente a evolução da mesma, procedendo sua ressecção cirúrgica apenas se houver alteração em seu comportamento ou aparecimento de algum sintoma ${ }^{4,5}$. Porém, nem sempre é possível diferenciar tumores benignos e malignos no pré-operatório ${ }^{6}$, por este motivo, se houver dúvidas, ou se o tumor for muito grande, muitas vezes ele deverá ser ressecado, mesmo que seja benigno ${ }^{5}$. Embora as metástases hepáticas de outras neoplasias possam ser submetidas a ressecção em cunha, ou até a lobectomia hepática, estes são procedimentos de exceção, a serem adotados em casos selecionados. Outra indicação de hepatectomia parcial são casos de laceração extensa do fígado, em que não se consegue fazer uma adequada hemostasia ${ }^{8-10}$.

A hepatectomia parcial é um procedimento cirúrgico tecnicamente difícil, que exige uma cuidadosa dissecção das estruturas do hilo hepático, das veias supra-hepáticas e, muitas vezes, da veia cava inferior em sua porção retro-hepática. A hemostasia deve ser feita de maneira rigorosa, pois a presença de uma neoplasia, mesmo que benigna, faz com que a vascularização hepática aumente consideravelmente, havendo com freqüência numerosos vasos neoformados calibrosos e de parede frágil, que sangram facilmente (Figura-3). Deve-se ter cuidado excepcional com a hemostasia, principalmente em recém-nascidos ou crianças de baixo peso. Nestes pacientes, sangramentos pequenos, porém contínuos, ao longo de uma cirurgia prolongada, vão se somando até representarem uma fração não desprezível da volemia, exigindo muitas vezes que a criança receba transfusão para reposição das perdas durante o ato cirúrgico.

Nas hepatectomias, mais do que em outras cirurgias mais comuns, é fundamental que o cirurgião conheça profundamente a anatomia da região a ser 
operada, pois pequenos erros podem levar a graves conseqüências. O cirurgião deve estar preparado para identificar possíveis variações anatômicas e, sempre que necessário, modificar a técnica cirúrgica em decorrência dos achadosintra-operatórios.

Alguns clássicos cuidados não devem ser esquecidos ao se realizar uma lobectomia hepática e estas recomendações, que nada mais são que um reforço no apuro com que deve ser feita a investigação clínica e o procedimento cirúrgico, servem como uma intenção de protocolo básico:

1)- A indicação cirúrgica deve ser precisa. Deve-se evitar a tentativa de ressecção de extensos tumores que infiltram mais de um lobo hepático, sem antes procurar reduzi-los através de quimioterapia pré-operatória. Da mesma forma, muitos tumores benignos podem ser retirados através de cirurgias menos agressivas, ou até acompanhados clinicamente, reservando-se as lobectomias para casos em que isto não seja possível.

2)- A investigação diagnóstica préoperatória deve ser feita de maneira cuidadosa, com radiografias contrastadas, ultra-sonografia, tomografia computadorizada e, em alguns casos, ressonância magnética. Assim o cirurgião terá uma idéia exata do tamanho e localização do tumor, bem como avaliará com segurança a possível existência de infiltração em estruturas contíguas ou de metástases à distância. As arteriografias seletivas podem e devem ser dispensadas, principalmente em crianças pequenas, por serem um procedimento invasivo que nem sempre traz informações indispensáveis ao cirurgião.

3)- A veia supra hepática direita ou esquerda, que drena o lobo hepático a ser ressecado, sempre que possível deve ser ligada antes de se iniciar a secção do parênquima. Isto diminuirá a hemorragia que ocorre retrogradamente, mesmo após a ligadura dos vasos do hilo hepático. Apenas quando isto não for possível, a veia será então ligada por dentro do parênquima hepático, durante a secção do fígado, no momento em que for identificada.

4)- A veia supra hepática média situa-se exatamente ao longo do plano anatômico que divide o fígado em lobos direito e esquerdo, e drena partes de ambos os lobos. Deve, portanto, ser preservada com cuidado sempre que a ressecção hepática for realizada através da secção deste plano. $\mathrm{O}$ cirurgião deve ligar apenas os ramos que provêm do lado do fígado que será retirado, preservando os ramos provenientes do outro lobo e o tronco principal da veia.

5)- O ramo esquerdo da veia porta tem parte de seu trajeto no plano que separa o segmento lateral do segmento medial do lobo esquerdo do fígado. Este trecho da veia segue após a inserção do ligamento redondo $-\mathrm{a}$ antiga veia umbilical - levando sangue a ambos os segmentos do lobo esquerdo hepático. Devem ser, portanto, ligados apenas os ramos que se dirigem ao segmento lateral (no caso de estar sendo realizada uma segmentectomia lateral esquerda), ou os ramos que se dirigem ao segmento medial (no caso de estar sendo realizada uma trissegmentectomia hepática) (Figura-4).

6)- Durante a secção do parênquima hepático, bem como durante qualquer outro passo cirúrgico em que haja a possibilidade de abertura acidental de alguma veia de grande calibre, o anestesista deve ser alertado para que mantenha a respiração do paciente com pressão expiratória final positiva (PEEP), diminuindo assim o risco de embolia gasosa.

7)- Como medida de segurança adicional, em casos difíceis, de tumores muito extensos, particularmente os do lobo direito, recomenda-se passar cadarços proximal e distalmente na veia cava inferior, não só para maior controle da hemostasia, mas também para facilitar sua rafia caso seja acidentalmente (ou intencionalmente) aberta.

8)- A drenagem da cavidade peritoneal após as hepatectomias parciais deve ser feita sempre e de maneira ampla, sendo um ponto importante na recuperação pós-operatória, diminuindo a incidência de hematomas e abscessos e facilitando o controle e tratamento de eventuais fístulas biliares.

9)- A superfície cruenta do fígado seccionado deve ser peritonizada ou suturada, o que diminui o sangramento pós-operatório e a probabilidade de ocorrer uma fístula biliar.

10)- Finalmente, o cirurgião e o anestesista devem estar preparados para a possibilidade de a laparotomia inicialmente planejada transformar-se em uma toraco-laparotomia, principalmente em crianças maiores, nas quais o fígado é maior e a arcada costal é menos maleável.

Em relação à indicação cirúrgica da lobectomia hepática nos casos de tumores benignos, acreditamos que merece ser revista a conduta 


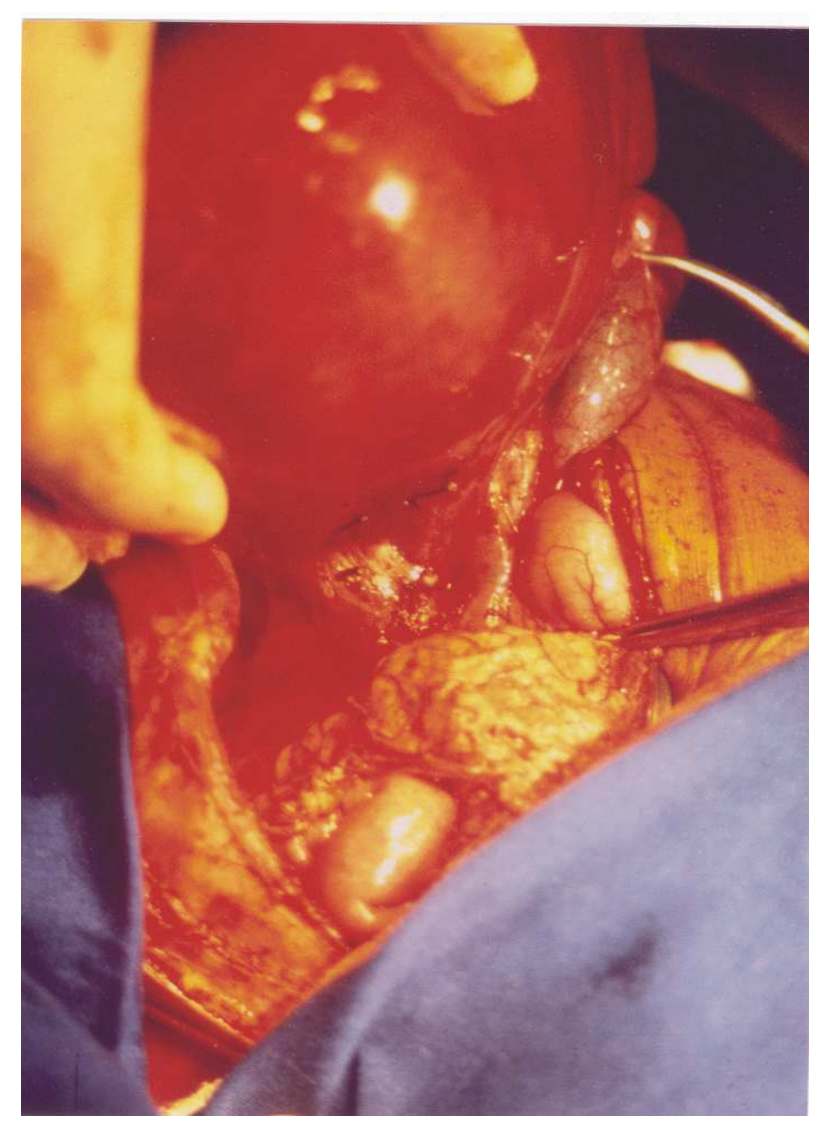

Figura 1 - Detalhe da ligadura dos ramos da veia cava inferior

muitas vezes adotada para os pacientes portadores de hemangioendotelioma. Alguns autores recomendam conduta expectante ${ }^{11}$, outros recomendam operar estes casos apenas quando forem sintomáticos ${ }^{12}$. Numerosas revisões,

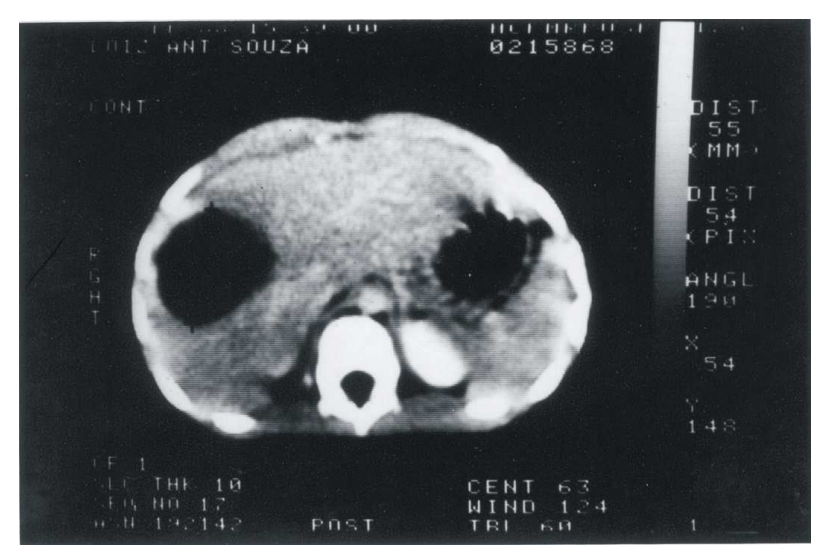

Figura 2 - Cavidade intra-hepática conseqüente a trauma abdominal fechado, causando hemobilia

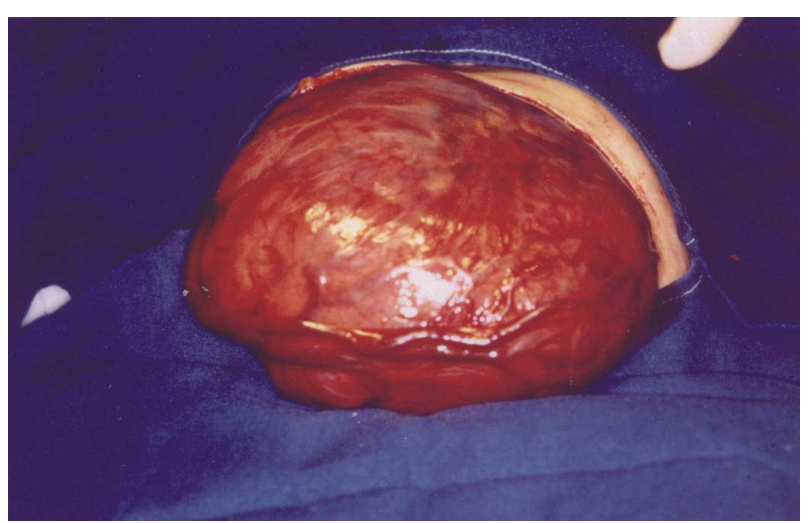

Figura 3 - Tumor hepático em recém-nascido, com neoformação vascular

entretanto, mostram que um número muito grande destes pacientes evolui para insuficiência cardíaca grave à medida que o tumor aumenta de tamanho (Tabela-1). Estes casos são resistentes ao tratamento clínico e freqüentemente necessitam ser

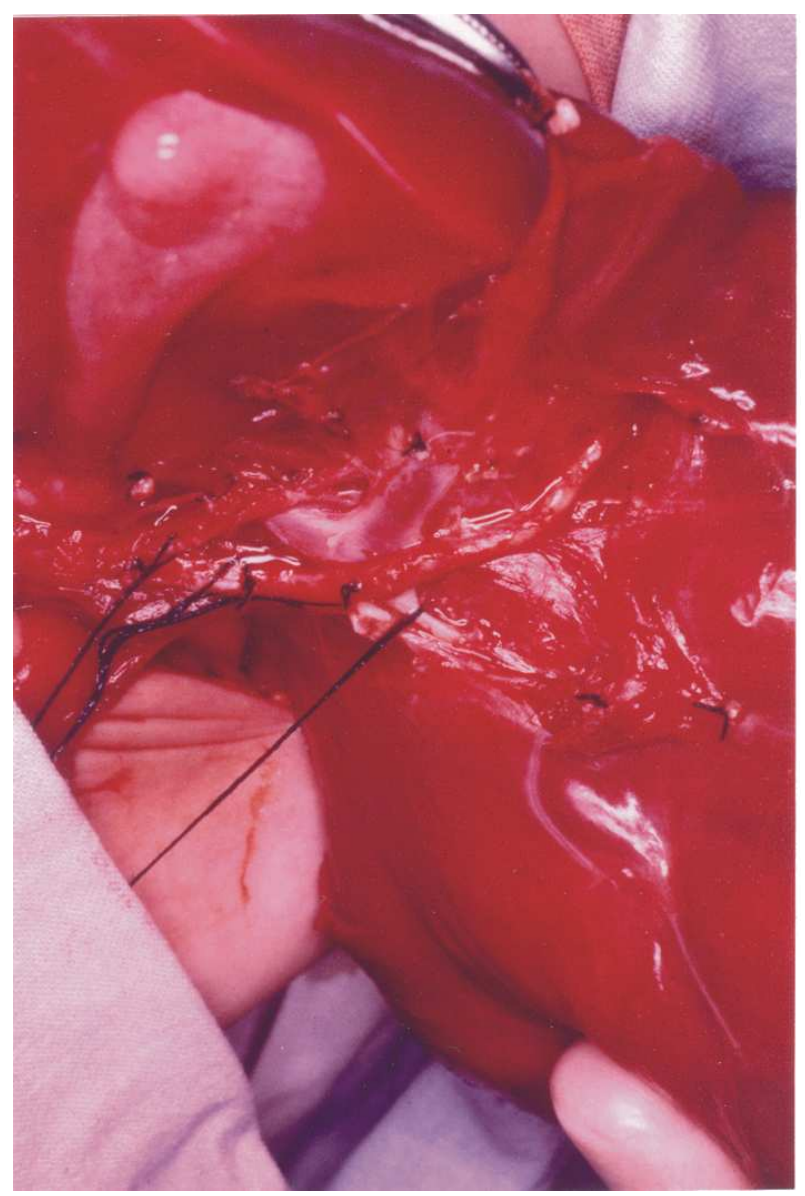

Figura 4 - Dissecção dos ramos arteriais e venosos para o lobo esquerdo do fígado 
submetidos a cirurgia, mesmo sem adequadas condições metabólicas e hemodinâmicas, o que implica em maior morbidade e mortalidade. Tendo em vista a alta incidência desta complicação ${ }^{6,7,13-19}$, e a decorrente necessidade de se submeter a criança a uma cirurgia de grande porte, em caráter de urgência, em precárias condições gerais e clinicamente instável, recomendamos que os hemangioendoteliomas hepáticos devam ser operados eletivamente antes de apresentarem tais sintomas.

Becker ${ }^{16}$, fazendo uma extensa revisão de 119 relatos de hemangioendotelioma hepático em crianças, encontrou uma incidência de insuficiência cardíaca congestiva de $35 \%$ nos casos de tumor unifocal e $68 \%$ nos casos de tumor multifocal. A mortalidade total nos pacientes em que se optou por tratamento clínico foi de $60 \%$. Considerando-se apenas os casos passíveis de tratamento cirúrgico, isto é, os tumores unifocais, houve uma mortalidade de $33 \%$ nos casos não operados, contra $8 \%$ nos operados. Outros autores relatam índices ainda maiores de insuficiência cardíaca congestiva (Tabela 1). Holmin ${ }^{7}$ enfatiza o fato de que o prognóstico das crianças com tumores benignos de fígado é sempre bom, exceto nos portadores de hemangioendotelioma. Estes dados parecem indicar que o fato de tais tumores poderem apresentar regressão espontânea não justifica a opção pela conduta expectante. A morbidade e a mortalidade decorrente do tratamento clínico fazem com que se justifique a indicação de uma hepatectomia, sempre que o tumor for considerado ressecável.

Estes números revelam também a baixa incidência deste tipo de tumor, com casuísticas em grandes hospitais tendo em média menos de 15 casos (Tabela-1). No presente estudo, as cinco crianças portadoras de hemangioendotelioma foram submetidas a cirurgia, todas antes de apresentarem sinais de descompensação cardíaca. Houve boa evolução pós-operatória em $100 \%$ dos casos.

Outra indicação cirúrgica que merece discussão é a hemobilia. Esta é uma condição rara em crianças e, na grande maioria dos casos, pode ser resolvida com medidas conservadoras, como a embolização através de arteriografia seletiva. Em casos de falha deste tratamento, pode-se recorrer a debridamento ou curetagem da cavidade intrahepática, ou ligadura seletiva da artéria hepática. Apenas em casos excepcionais, como o paciente apresentado nesta casuística, no qual havia numerosas cavidades de grande volume dentro do parênquima hepático, e após falha dos outros procedimentos terapêuticos, pode-se optar pela lobectomia hepática. Deve-se enfatizar, no entanto, que este não é o tratamento de escolha e deve ser reservado como último recurso em casos selecionados.

A casuística aqui relatada mostrou que os doze pacientes submetidos a hepatectomia parcial em nosso serviço apresentaram evolução excepcionalmente boa. Não houve nenhum caso de hemorragia pós-operatória, fístula biliar, abscesso intra-peritoneal, ou infecção da ferida cirúrgica. Todos os pacientes apresentaram adequada função hepática pós-operatória, sem qualquer sinal ou sintoma, clínico ou laboratorial, de insuficiência hepática. Exceto pela necessidade de transfusão sanguiínea em $70 \%$ dos pacientes para repor as perdas intra-operatórias, não houve qualquer complicação durante $o$ ato cirúrgico.

Tabela 1. Incidência de insuficiência cardíaca congestiva (ICC) e óbito nos pacientes com hemangioendotelioma hepático.

\begin{tabular}{|c|c|c|c|c|c|}
\hline Autor & Pacientes & ICC & Porcentagem & Óbito & Porcentagem \\
\hline Larcher, $1981^{13}$ & 5 & 4 & 80 & & \\
\hline Ehren, $1983^{14}$ & 29 & & & 4 & 14 \\
\hline Holcomb, $1988^{15}$ & 16 & 7 & 44 & 3 & 19 \\
\hline Becker, $1989^{16}$ & 95 & & & 38 & 60 \\
\hline Fellow, $1991^{17}$ & 6 & 5 & 83 & & \\
\hline Luks, $1991^{18}$ & 16 & 9 & 56 & & \\
\hline Davenport, $1995^{19}$ & 11 & 10 & 91 & 2 & 18 \\
\hline Von Schweinitz, $1995^{6}$ & 17 & 7 & 41 & & \\
\hline
\end{tabular}


Pode-se, portanto, concluir que, embora as lobectomias hepáticas sejam procedimentos cirúrgicos difíceis e que exigem grande experiência técnica e apurado conhecimento teórico ${ }^{1}$, não há necessidade de sofisticados recursos tecnológicos para serem realizados ${ }^{20}$. Tais recursos são, sem dúvida, úteis, e é indiscutível que facilitam o ato cirúrgico, principalmente em casos de tumores muito extensos. Porém não são indispensáveis, e o cirurgião, trabalhando em hospitais equipados apenas com recursos mais tradicionais, pode obter excelentes resultados a curto e a longo prazo no tratamento dos tumores hepáticos.

\begin{abstract}
Background: Although less frequent in childhood nowadays, hepatectomies may still be the best choice in some cases. Our objective is to describe a 10-year experience with large hepatic resections in pediatric patients. Methods: A retrospective review of clinical and surgical data of 12 patients submitted to hepatic lobectomy, at Pediatric Surgery Service of Hospital das Clinicas da Faculdade de São Paulo and Hospital Santa Lydia, Ribeirão Preto (Brazil), between 1985 and 1995. Results: There were eight left lobectomies and four right lobectomies. The indication of surgery were neoplastic diseases in 10 children, and complicated hepatic trauma in two. The age range was six days to 16 months in children with hepatic tumors, and the mean age was 3,8 months. Histopathological diagnosis were hemangioendothelioma in five (50\%), hepatic adenoma in two (20\%), hepatoblastoma in two (20\%), and hepatic carcinoma in one (10\%). Mean tumor weight was 7,1\% of body weight. Mean surgical time was $2 \mathrm{~h}$ $58 \mathrm{~m}$. Postoperative follow-up was up to 141 months, and mean follow-up was 76,5 months. Seven patients received intraoperative blood transfusion, corresponding to 23,3\% of volemy. Only one patient had a late reoperation for resection of a new tumor. There were no postoperative complications. Conclusion: Although hepatic lobectomy is a major surgery, and undoubtedly technically difficult, it can be safely performed without expensive devices and sophisticated methods (some of them not always available in common hospitals) if the surgeon is skilled and well prepared for such a procedure.
\end{abstract}

Key words: Hepatectomy; Hepatic Tumors; Child; Infant

\section{REFERÊNCIAS}

1. Meyer D, Christie P - Liver resections in Auckland; a Retrospective Review of Patients 1986-1991. NZ Med J, 1995;108:151-152.

2. Couinaud $\mathrm{C}$ - Lobes et segments hépatiques. Notes sur l'architecture anatomique et chirurgicale du foie. Presse Med, 1954, 62:709-712.

3. Couinaud C - Bases anatomiques des hépatectomies gauche et droite reglées: techniques qui en découlent. J Chir, 1954,70:933-966.

4. Reymond D, Plaschkes J, Luthy AR, et al. - Focal nodular hyperplasia of the liver in children: review of follow-up and outcome. J Pediatr Surg, 1995, 30:1590-1593.

5. Hutton KA, Spicer RD, Arthur RJ, et al . - Focal nodular hyperplasia of the liver in childhood. Eur J Ped Surg, 1993, 3:370-372.
6. Von Schweinitz D, Gluer S, Mildenberger H-Liver tumors in neonates and very young infants: diagnostic pitfalls and therapeutic problems. Eur J Pediatr, 1995, 5:72-76.

7. Holmin T, Kullendorf CM - Diagnostic aids for benign tumors of the liver in children. Acta Paediatr Scand, 1981, 70:755-757.

8. Feliciano DV, Mattox KL, Jordan GL Jr - Intra-abdominal packing for control of hepatic hemorrhage: a reappraisal. J Trauma, 1981, 21:285-290.

9. Krige JE, Worthley CS, Terblanche J - Severe juxta-hepatic venous injury: survival after prolonged hepatic vascular isolation without shunting. HPB Surgery, 1990, 3:39-45.

10. Hollands MJ, Little JM - The role of hepatic resection in the management of blunt liver trauma. World J Surg, 1990, 14:478-482.

11. Rosen PR, Mewborne EB, Macpherson RI, et al. Hepatic benign vascular tumor in infancy: correlative imaging. Clin Pediatr, 1982, 21:180-182. 
12. Hobbs KE - Hepatic hemangiomas. World J Surg, 1990,14:468-471.

13. Larcher VF, Howard ER, Mowat AP - Hepatic haemangiomata: diagnosis and management. Arch Dis Child, 1981;56:7-14.

14. Ehren H, Mahour GH, Issacs Jr, H - Benign liver tumors in Infancy and childhood: report of 48 cases. Am J Surg, 1983,145(3):325-329.

15. Holcomb GW, O’Neill JA Jr, Mahboubi S, et al. - Experience with hepatic hemangioendothelioma in infancy and childhood. J Pediatr Surg, 1988, 23:661666.

16. Becker JM, Heiler MS - Hepatic hemangioendotheliomas in infancy. Surg Gynecol Obstet, 1989,168:189-200.

17. Fellow KE, Hoffer FA, Markowitz RI, et al. -Multiple collaterals to hepatic infantile hemangioendotheliomas and arteriovenous malformations: effect of embolization. Radiology, 1991, 181(3):631-632.

18. Luks FI, Yazbeck S, Brandt ML, et al. - Benign liver tumors in children: a 25-year experience. J Pediatr Surg, 1991, 26:1326-1330.

19. Davenport M, Hansen L, Heaton ND, et al. - Hemangioendothelioma of the liver in infants. J Pediatr Surg, 1995, 30:44-48.

20. Hata Y, Sasaki F, Takahashi H, et al. - Liver resection in children, using a water-jet. J Pediatr Surg, 1994, 29:648-650.

Endereço para correspondência:

Roberto Cardoso dos Santos

Rua Quintino Bocaiúva, 1105

14015-160 - Ribeirão Preto - SP 